\title{
Microbots decorated with silver nanoparticles kill bacteria in aqueous media
}

D. Vilela $\uparrow$, M.M. Stanton $\dagger$, J. Parmart+ and S. Sanchez $\dagger+\phi$

$\dagger$ Max-Planck Institute for Intelligent Systems, Heisenbergstr. 3, 70569 Stuttgart, Germany. E-mail: sanchez@is.mpg.de;

t. Institute for Bioengineering of Catalonia (IBEC), Baldiri I Reixac 10-12, 08028 Barcelona, Spain. Email: ssanchez@ibecbarcelona.eu

$\phi$ Institució Catalana de Recerca i Estudis Avançats (ICREA), Psg. Lluís Companys, 23, 08010 Barcelona, Spain

KEYWORDS Silver nanoparticles, water propulsion, bacteria remediation, biocompatibility and environmental application.

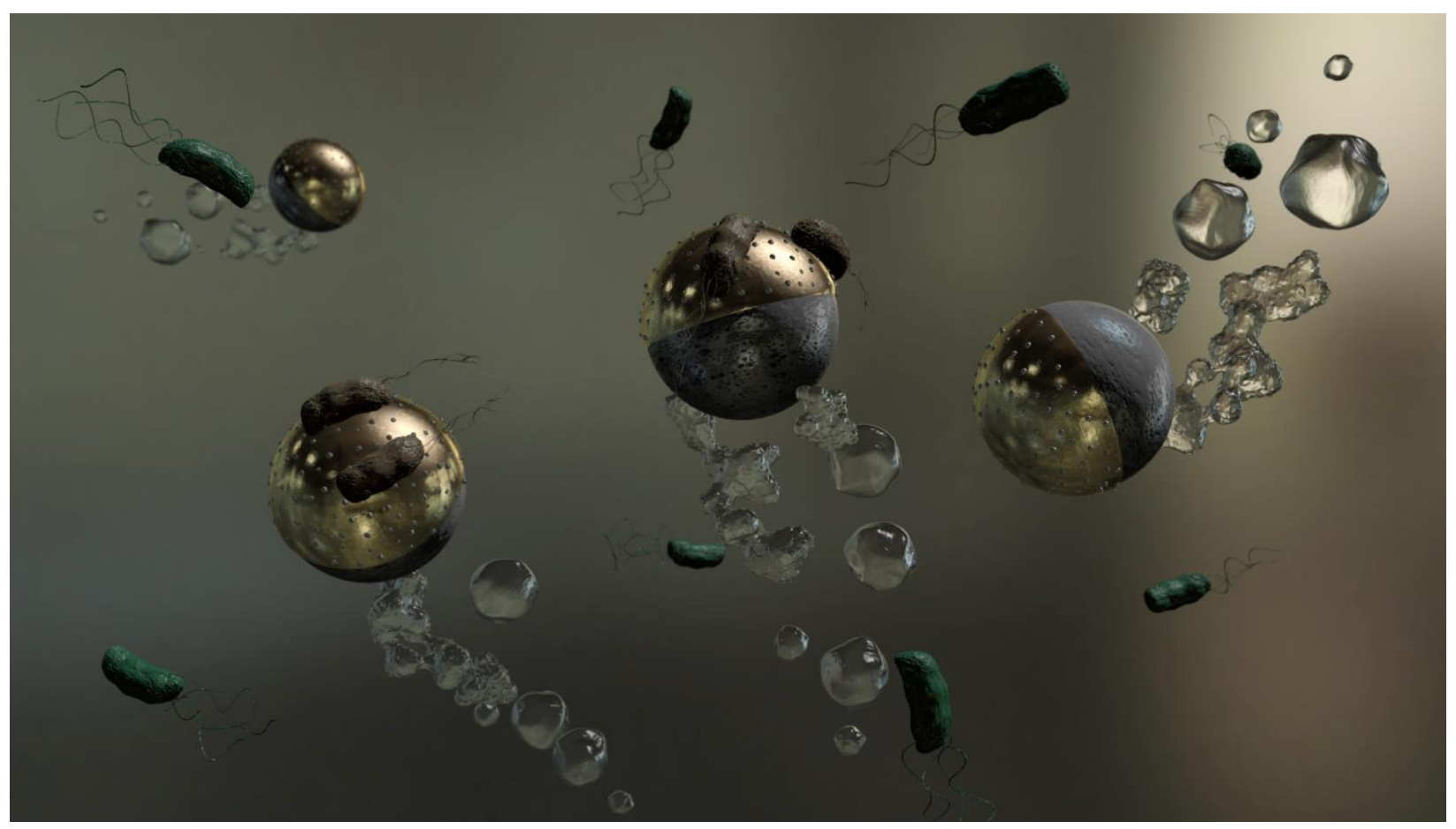

Water contamination is one of the most persistent problems in public health. The resistance of some pathogens to conventional disinfectants can require the combination of multiple disinfectants or increased their dosages which may produce harmful by-products. Here, we describe an efficient disinfection and removal method of Escherichia coli (E. coli) from contaminated water by using 
water self-propelled Janus microbots decorated with silver nanoparticles (AgNPs). The spherical Janus microbot's structure consists of a magnesium $(\mathrm{Mg})$ microparticle as a template that also functions as propulsion source by producing hydrogen bubbles while in contact with water, an inner iron $(\mathrm{Fe})$ magnetic layer for their remote guidance and collection, and an outer AgNP coated gold $(\mathrm{Au})$ layer for bacteria adhesion and bactericidal properties. The active motion of microbots improves the chances of the contact of microbot surface decorated AgNPs with the bacteria, which provokes the selective $\mathrm{Ag}^{+}$release in bacteria cytoplasma, and their self-propulsion increase diffusion of the released $\mathrm{Ag}^{+}$ions. In addition, the AgNPs coated Au metal cap of the microbots has dual capabilities capturing bacteria and then killing them. Thus, we have demonstrated that AgNPs coated Janus microbots are capable of efficiently killing more than $80 \%$ of E. coli compared to colloidal AgNPs that killed less than 35\% of E. coli in 15 minutes in contaminated water solutions. After bacteria capture and extermination, the magnetic properties of the cap allow microbots to be collected from water with the captured dead bacteria, leaving water with no contaminants. The presented biocompatible Janus microbots offers an encouraging method for rapid disinfection of water. 


\section{Introduction}

One of the most persistent problems affecting people throughout the world is inadequate access to clean water. In particular, waterborne diseases can have a devastating effect on public health. ${ }^{1}$ The current disinfection methods use chemical (free chlorine, chloramines and ozone) ${ }^{2}$ and physical disinfectants (UV-light, ${ }^{3,4}$ electronic radiation ${ }^{5}$ and heat ${ }^{6}$ ). However, the resistance of some pathogens to conventional disinfectants requires the combination of these disinfection methods or higher disinfectant dosages ${ }^{7}$ which can react with constituents in natural water forming harmful disinfection by-products. ${ }^{8}$ Therefore, there is an urgent need to develop more effective, innovative, low-cost, robust water cleaning methods, which do not adversely affect the environment or damage human health.

In recent years, catalytic self-propelled micro- and nanomotors have demonstrated diverse practical applications within the environmental field. ${ }^{9}$ These micromotors convert chemical energy into motion which enhances the micromixing and mass transfer in the solutions. ${ }^{10-12}$ In addition, they contain many surface functionalities ${ }^{13}$ which open new opportunities to develop micro and nanodevices as efficient tools for water cleaning. ${ }^{14-16}$ Currently, micromotors have been developed for the efficient degradation of organic pollutants, ${ }^{12,17-20}$ chemical warfare agents ${ }^{11,21}$ and the capture of organic and inorganic ${ }^{22-29}$ pollutants, such as heavy metals. ${ }^{29}$ New bactericidal micromotors $^{30-32}$ are recently being developed as new efficient tools for cleaning waterborne bateria due to the increasing threat of antibiotic resistant bacteria and the harmful chemical byproducts generated by conventional water disinfection methods. ${ }^{8}$

Alternative methods of bacteria disinfection have utilized silver: silver has been commonly used in the form of metallic silver and silver ions for the treatment of injuries, wounds, and bacterial infections. ${ }^{33}$ Currently, due to the fast growth of nanotechnology, silver nanoparticles (AgNPs) are 
widely used in medical ${ }^{34}$ and consumer ${ }^{35}$ products because of their potent bactericidal effect. ${ }^{36-40}$ AgNPs have an exceptionally large surface area which improves the contact with microorganisms and provides efficient antimicrobial properties compared to other Ag salts. ${ }^{39}$ Furthermore, AgNPs can release $\mathrm{Ag}^{+}$ions at a desired rate and location depending on their designs (size, surface coatings, etc...). Thus, AgNPs can be used as the vehicle to efficiently deliver $\mathrm{Ag}^{+}$ions into bacteria cytoplasma due to the localized acidic $\mathrm{pH}$ of a bacteria membrane $\mathrm{e}^{41,42}$ enchances the rate of $\mathrm{Ag}^{+}$ion release from $\mathrm{AgNPs}$ adhered to bacteria. ${ }^{36} \mathrm{Ag}$ is able to bind to thiol groups present in proteins disrupting their functionality by compromising the permeability of the bacteria membrane leading to cell lysis and death. ${ }^{36,43}$

Here, we present an efficient disinfection and removal of bacteria from contaminated water by using silver nanoparticle (AgNPs) coated Janus microbots self-propelled by the reaction of magnesium $(\mathrm{Mg})$ in water producing hydrogen $\left(\mathrm{H}_{2}\right)$ bubbles. ${ }^{44,45}$ The high antibacterial capacity of the developed AgNPs based Janus microbots is the result of two synergistic effects: (i) the enhanced contact killing by the combination of the Janus microbot self-propulsion with the immobilized AgNPs on their surface ${ }^{46}$ and (ii) the capability of gold bacteria adhesion. ${ }^{47,48}$ Therefore, the AgNPs coated Janus microbots are able to clean waterborne bacteria within 15 min of swimming in contaminated water. In addition, AgNPs coated Janus microbots can eliminate bacteria by their attachment to Au surface using a magnetic field. After microbot swimming and bacteria harvest, the magnetic field can be used to remove the microbots and captured dead bacteria. Unlike previous reports of microswimmers using toxic fuel sources such as $\mathrm{H}_{2} \mathrm{O}_{2}$, the Mg-based Janus microbot is biocompatible, environmentally friendly, and does not produce chemical waste during and after operation. Thus, the combination of active systems and 
nanomaterials to develop new micro- and nanomotors can open new horizons for demanded medicinal, energy and environmental applications.

\section{Results and discussion}

In our report, AgNPs coated Janus microbots are presented as an active bactericidal tool for water cleaning. These microbots are based on four materials: (1) Magnesium (Mg) microparticles (15 \pm 5 $\mu \mathrm{m})$ as a template structure and for self-propulsion, (2) iron (Fe) Janus cap which provides magnetic control, (3) gold ( $\mathrm{Au}$ ) layer on top of Fe layer providing an easily modifiable surface and cell adhesion and (4) AgNPs attached on the Au surface providing antibacterial properties to the microbots.

The detailed fabrication of the water-propelled AgNPs coated Janus microbots is described in Scheme 1. As shown, the microbots' fabrication is carried out by the consequent deposition of Fe and $\mathrm{Au}$ on a monolayer of previously cleaned commercial $\mathrm{Mg}$ microparticles using an e-beam metal evaporator (Scheme 1A). After the deposition, Janus microparticles were dispersed in an ethanol solution for the chemical modification of the Au surface using a solution of cysteamine hydrochloride. Then, AgNPs immobilization is mediated by overnight incubation of the cysteamine modified Janus microparticles in previously synthesized AgNPs solution (see

methods). ${ }^{49}$ A layer of AgNPs is formed on the Au surface of the Janus microparticles due to the interactions between AgNPs and amines (Scheme 1B). ${ }^{46,50}$ 


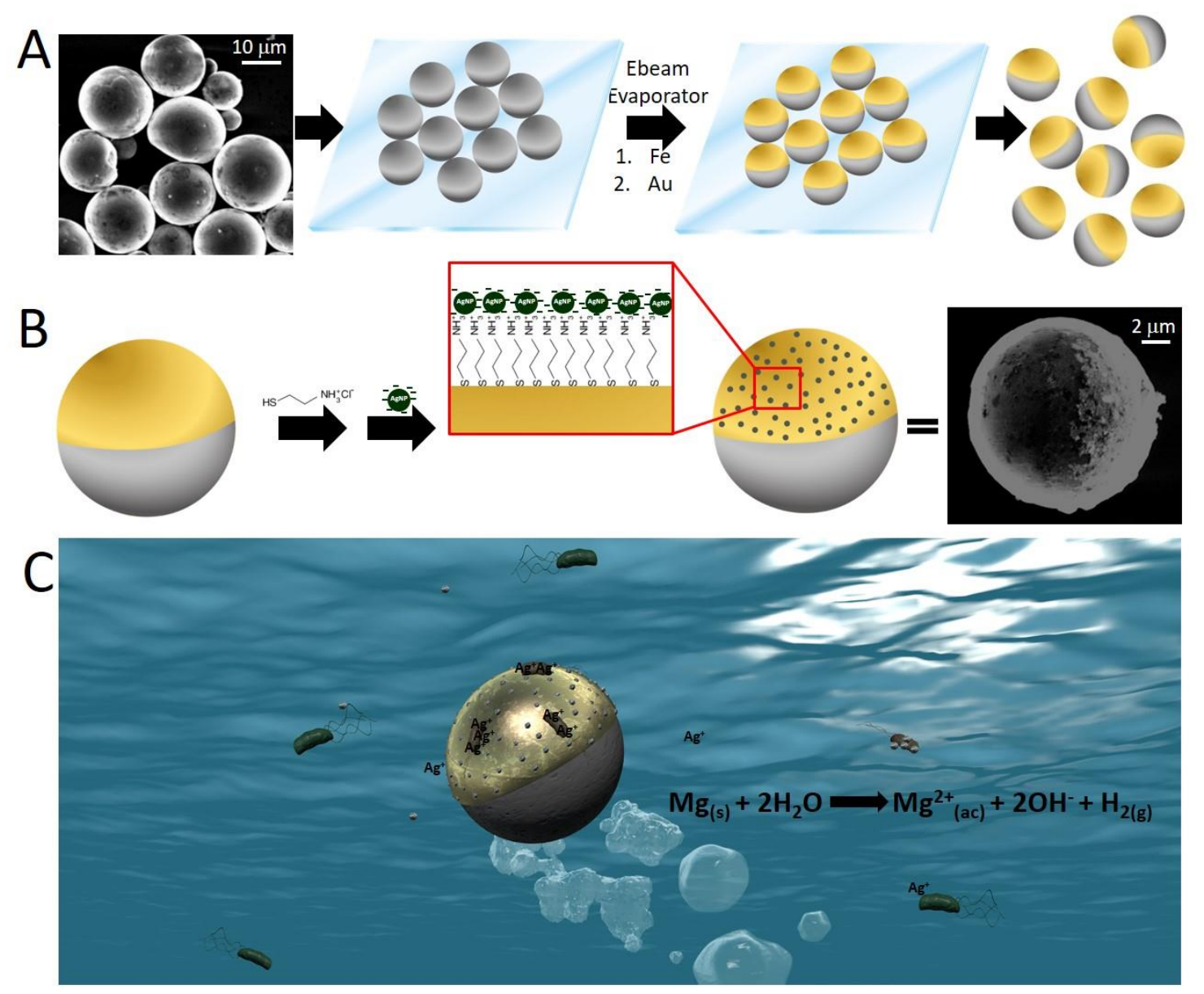

Scheme 1. Fabrication of the AgNPs coated Janus microbots. (A) A monolayer of $\mathrm{Mg}$ microparticles was deposited on a glass slide. An e-beam metal evaporator was used for the deposition of $14 \mathrm{~nm}$ layer of $\mathrm{Fe}$ and $35 \mathrm{~nm}$ layer of $\mathrm{Au}$. To obtain free Janus microbots, short ultrasound pulses in ethanol were applied. (B) Fabrication of the AgNPs coated Janus microbots by the modification of the Au surface first with cysteamine hydrochloride and then with previously synthesized AgNPs. ${ }^{49}$ (C) Schematic representation of the different possible mechanisms of bactericidal action caused by the water-propelled AgNPs coated Janus microbots.

The microbot Janus particle is composed of various materials for a multi-functional, environmental remediation system. The $\mathrm{Mg}$ microparticle reacts with water producing $\mathrm{Mg}$ ions $\left(\mathrm{Mg}^{2+}\right)$, hydroxide ions $\left(\mathrm{OH}^{-}\right)$and hydrogen gas $\left(\mathrm{H}_{2}\right)$ providing a microbubble propulsion mechanism which exerts enough force that enables the microbots to swim. The reaction of $\mathrm{Mg}$ with water is an spontaneous 
redox reaction in which $\mathrm{Mg}$ is oxidezed and $\mathrm{H}^{+}$prontons from water molecules are reduced to $\mathrm{H}_{2}$ (Scheme 1C). The inner Fe layer offers the ability for a remote guidance and removal of the microbots using an applied magnetic field. The outer Au layer provides surface chemistry for thiol attachment and subsequent AgNPs modification by electrostatic interactions (Scheme 1B) and helps bacteria adhesion (Scheme 1C). The presence of the AgNPs on the Janus microbot provides the particles' bactericidal behavior.

Scheme 1C represents the possible mechanisms of bactericidal action caused by the waterpropelled AgNPs coated Janus microbots: (i) direct contact with immobilized AgNPs on coated microbots, (ii) contact with AgNPs that could be released from the AgNPs coated microbots into the solution as colloids, and (iii) mediated via silver ions released either from colloidal AgNPs in the solution or immobilized nanoparticles. ${ }^{36,43,46}$
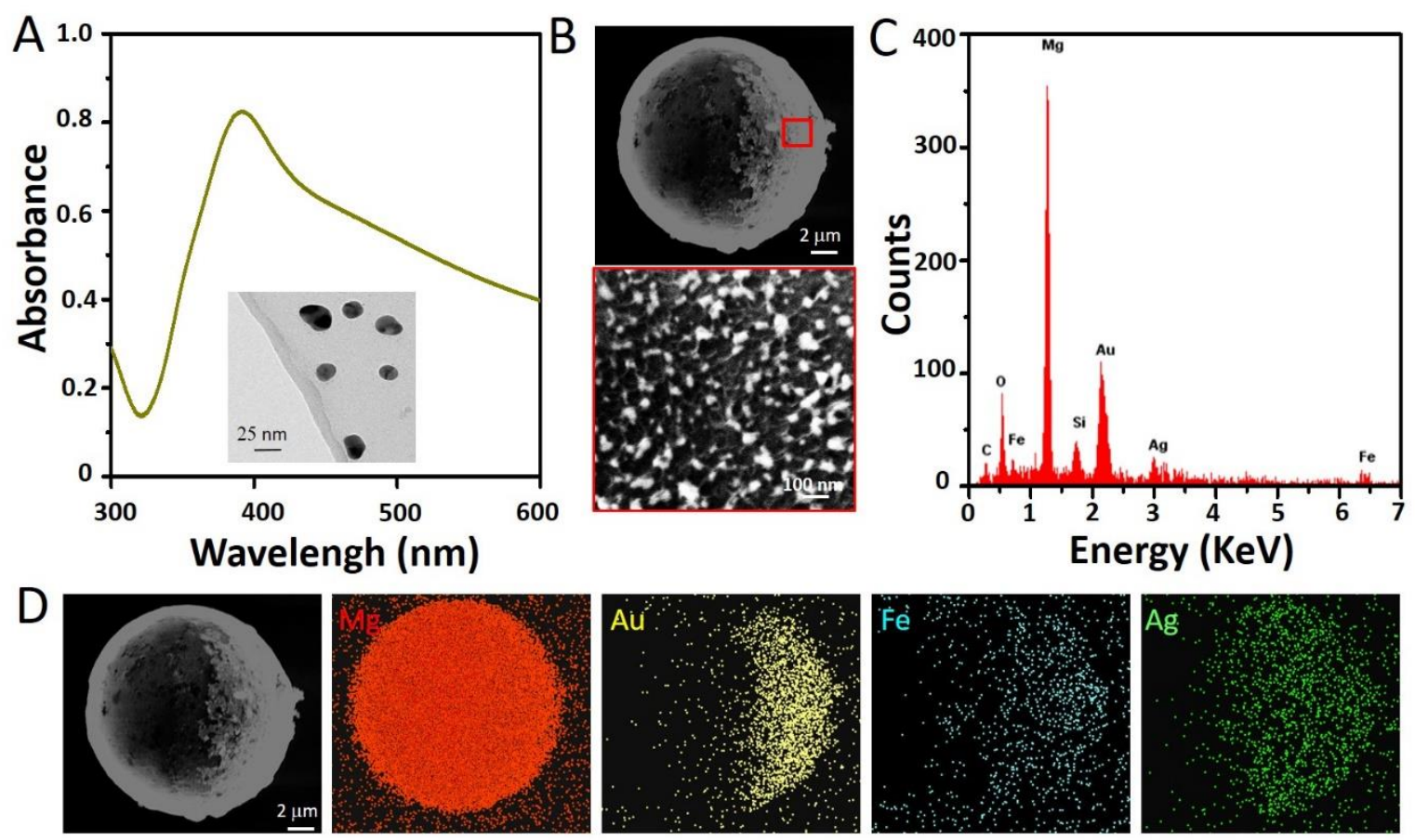

Figure 1. Chatacterization of colloidal AgNPs and AgNPs coated Janus microbots. (A) UV-visible spectrum of prepared AgNPs in colloidal solution (inset: TEM images corresponding to AgNPs in 
colloidal solution). (B) Top panel: SEM image of an AgNPs coated Janus microbot; bottom panel: an enlarged SEM image of the Au modified surface with AgNPs. (C) EDX element analysis of an AgNPs coated Janus microbot. (D) EDX mapping of the metallic elements of an AgNPs based Janus microbot.

Janus microbots were characterized by scanning electron microscopy (SEM), energy dispersive X-ray spectroscopy (EDX), UV-Vis spectroscopy, and transmission electronic microscopy (TEM) (Figure 1). Figure 1A displays the characteristic maximum absorbance peak of AgNPs colloidal aqueous solution at $398 \mathrm{~nm}$ in the UV-Visible spectrum. Additionally, the size of the AgNPs in colloidal solution is characterized by TEM (Figure 1A, inset) observing an average diameter of $23 \pm 6 \mathrm{~nm}$. Figure 1B, C and D illustrate the characterization of AgNPs coated Janus microbots using SEM and EDX. In the Figure 1B top image, an SEM image shows an AgNPs coated Janus particle, where the two faces of the particle can be differentiated. The bottom image is a magnified view of a small area of the AgNPs coated Au surface (red square) where AgNPs are visible. Figure 1C illustrates the EDX analysis which confirms the metallic components of the AgNPs coated Janus microbots, including $\mathrm{Mg}, \mathrm{Au}, \mathrm{Fe}$ and $\mathrm{Ag}$, where $\mathrm{Mg}$ is the major component. To prove how these elements are distributed in the Janus microbot structure, Figure 1D displays the mapping of the detected metallic elements performed by EDX analysis. As it can be observed, the bulk of the sphere is $\mathrm{Mg}$ since it is the base material of the particle. Only half of the particle, i.e. the Janus particle cap, contains Fe, Au and Ag. 

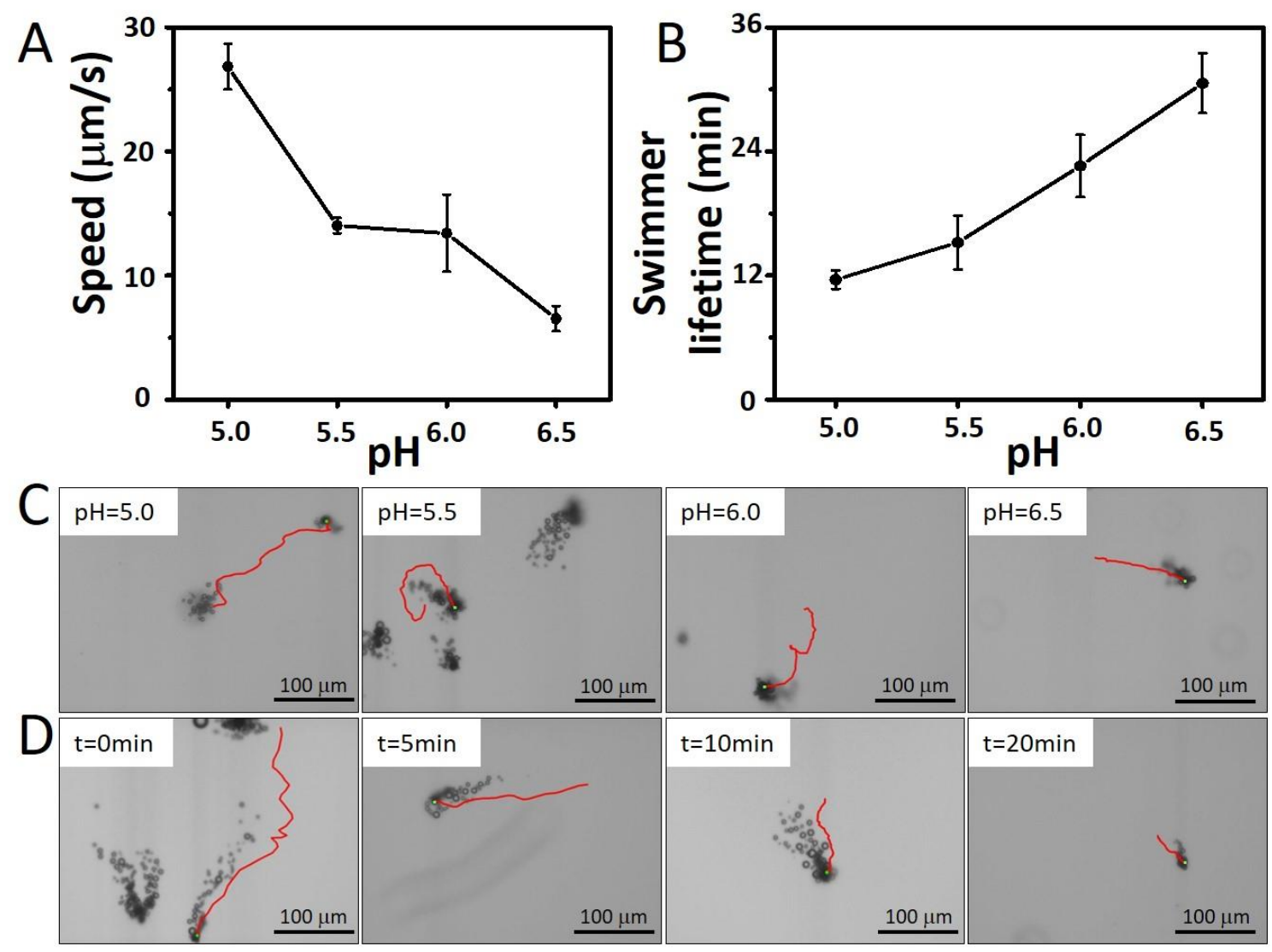

Figure 2. Swimming behavior of AgNPs coated Janus microbots: (A) Representation of the average velocities of Janus microbots vs. different $\mathrm{pHs},(\mathbf{B})$ representation of the average swimmer lifetime vs. different pHs, (C) an AgNPs coated Janus microbot swimming over the pH (each image contains $10 \mathrm{~s}$ of tracking over $\mathrm{pH} 5,5.5,6$, and 6.5, respectively), (D) an AgNPs coated Janus microbot over their swimming lifetime (each image contains $10 \mathrm{~s}$ of tracking over $0,5,10$, and 20 min observation, respectively). Experimental conditions: PBS $(\mathrm{pH}=6.0)$ and $2 \%$ tween.

To prove the capability of the motion of AgNPs coated Janus microbots and consequently, their active behavior as bactericidal tools for water disinfectant, their swimming behavior is studied. Figure 2A shows the quantification of the speed of the Janus microbots against a short range of pHs $(5.0-6.5)$ of the swimming solution, similar to the $\mathrm{pH}$ of drinkig water. Figure $\mathbf{2 C}$ and Video S1 display the traveled distance of AgNPs coated Janus microbots at different pHs during 10 seconds. As the $\mathrm{pH}$ decreases, the speed of the microbots increases as well as their total distance 
traveled. This behavior is due to increased degradation rate of $\mathrm{Mg}$ in acidic aqueous solution, producing increased amounts of $\mathrm{H}_{2}$, and, as consequence, more bubbles for a greater selfpropulsion velocity. Thus, the highest speeds of the microbots are observed at $\mathrm{pH} 5.0$ $(26.9 \pm 1.8 \mu \mathrm{m} / \mathrm{s})$, followed by $\mathrm{pH} 5.5(14.0 \pm 0.6 \mu \mathrm{m} / \mathrm{s}), \mathrm{pH} 6.0(12.6 \pm 3.0 \mu \mathrm{m} / \mathrm{s})$ and lastly $\mathrm{pH} 6.5$ $(6.2 \pm 1.0 \mu \mathrm{m} / \mathrm{s})$. The constant consumption of Mg during the reaction limits the life time of the microbots (Figure 2D and Video S2) and is directly related to the $\mathrm{pH}$ of the solution. That fact is observed in Figure 2B, where swimming lifetime of the Janus microbots is shorter in acidic $\mathrm{pH}$ compared to microbots in a more neutral $\mathrm{pH}$. Addition of a biocompatible surfactant, such as Tween-20, made the Janus microbots swim more efficiently, improving their active movement and showing higher efficacy in water solution by reducing the surface tension and producing small bubbles for the propulsion. The speed of the microbots at different tween concentrations was studied (Figure S2). The speed of Janus microbots at pH 6 increased with increased concentration of Tween-20, but after $2 \% \mathrm{v} / \mathrm{v}$ of Tween-20, microbot speeds remained constant. Therefore, to reach a compromise, $\mathrm{pH} 6$ and $2 \%$ of Tween-20 were selected as optimal $\mathrm{pH}$ and surfactant concentration, respectively, and 15 minutes as effective swimming time to carry out the remediation of bacteria-contaminated water by AgNPs coated Janus microbots. 


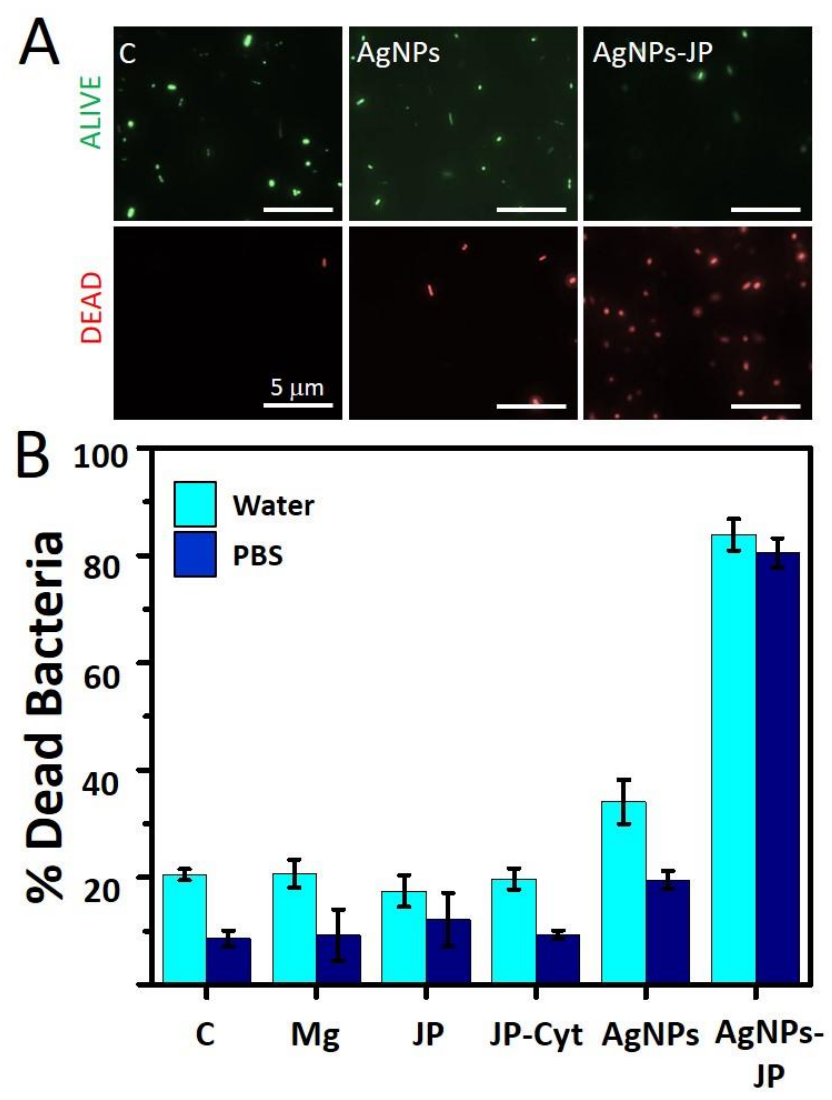

Figure 3. Bactericidal assay results using AgNPs coated Janus microbots. (A) Optical microscope images of bacteria using fluorescence lamp after bactericidal assays for control solution (C), AgNPs and AgNPs coated Janus microbots (AgNPs-JP). (Note that all the images have the same scale bar). (B) \% Dead E. coli bacteria. Experimental parameters and controls are compared in water and PBS. (E. coli, as model bacteria, is in contact with $0.5 \mathrm{mg}$ of $\mathrm{Mg}$ microparticles $(\mathrm{Mg})$, Janus microparticles (JP), Cysteamine modified JP (JP-Cyt) and AgNPs coated microbots (AgNPs-JP) for 15 min in water pH 6 (cyan) and PBS pH 6 (blue). All experiments were carried out at $2 \% \mathrm{v} / \mathrm{v}$ Tween-20.

To study the antibacterial capacity of AgNPs coated Janus microbots, E. coli are used as a model bacteria. Also, different control experiments are carried out demonstrating the efficiency of the active AgNPs coated Janus microbots approach. E. coli are one of the most well studied bacteria and are responsible for many bacterial infections in host organisms ingested from food or water sources being the best biological drinking water indicator for public health protection. ${ }^{51}$ 
Alternative methods are needed to remove these bacteria from water sources to ensure their drinkability. The AgNPs coated Janus microbots provide an ideal biocompatible platform for selfpropulsion, bacteria capture, bacteria termination, and removal of microbot and dead bacteria. After the microbots swam and killed bacteria in solution, they were removed and imaged using fluorescence viability studies and compared to their control groups. Figure 3 shows the antibacterial capability of microbots in comparison with control assays in PBS and water. The amount of particles were kept the same between each experimental group. The control group with only AgNPs, had the same quantity of AgNPs as the AgNPs coated Janus microbots since colloidal AgNPs amount adsorbed onto the AgNPs coated Janus microbots was estimated during the microbots fabrication process (Figure 1S). The same quantity (w/v) of $M g$ microparticles was compared to the $\mathrm{Au} / \mathrm{Fe} / \mathrm{Mg}$ Janus particles (JP), and the cysteamine modified $\mathrm{Au} / \mathrm{Fe} / \mathrm{Mg}$ Janus microparticles (JP-Cyt). The groups were studied in the presence of two different medias, water and PBS, at same $\mathrm{pH}$. As expected, Mg and Janus microparticles(JP and JP-Cyt) are not able to kill bacteria since they do not contain AgNPs. In addition, the hydrogen and ions liberated from the reaction of magnesium with water from these structures does not affect significantly the viability of the bacteria (Figure 3B, Mg, JP and Cyt-JP)). Static colloidal AgNPs are also employed as a control, since, as it is well-known, AgNPs interact with the outer membrane of bacteria, causing structural damage that leads to their death. ${ }^{36-40,43}$ Figure 3, also displays the percentage of dead E. coli in presence of AgNPs colloidal solution. AgNPs were able to kill around $25 \%$ of bacteria in PBS and less than $40 \%$ in water. This is due to the fact that $E$. coli prefer and have higher viability in salt solutions such as PBS. Incubation of $E$. coli in salt free water induces stress on bacteria making them easier to terminate. This indicates the presented motors would be highly effective at eliminating bacteria in drinking water that typically has low salt concentrations. 
The interaction of swimming bacteria with $\mathrm{AgNPs}$ and liberated $\mathrm{Ag}^{+}$ions in water solutions lead to increased bactericidal activity. However, the bigger bactericidal effect is observed when the bacteria are in contact with AgNPs coated Janus microbots which are able to kill more than $80 \%$ of $E$. coli. These microbots swim actively in water solution at $\mathrm{pH} 6$ for more than $15 \mathrm{~min}$ (Figure 2B) promoting an active contact of AgNPs with bacteria and the diffusion of $\mathrm{Ag}^{+}$ions, increasing the bactericidal efficiency of the AgNPs (Scheme 1C). The metallic ions released in solution after AgNPs and AgNPs coated Janus microbots were utilized for bacteria removal (Figure 4S, A and C) have been analyzed and compared with the ions released from AgNPs coated Janus microbots that were not exposed to E. coli. (Figure 4S, B). It is observed that the higher amount of released ions corresponds to $\mathrm{Mg}^{2+}$ (less than $20 \mu \mathrm{g} / \mathrm{mL}$ ) since the metallic Mg structure is consumed during time in contact with aqueous solution. $\mathrm{Ag}^{+}$ion concentration in solution was 3 -folds higher for AgNPs $(0.024 \pm 0.006 \mu \mathrm{g} / \mathrm{mL})$ than for the microbots $(0.007 \pm 0.001 \mu \mathrm{g} / \mathrm{mL})$ in the presence of bacteria. However, when the AgNPs coated microbots swam without presence of bacteria, the release was 10 -folds higher than the analogous assay with bacteria $(0.07 \pm 0.02 \mu \mathrm{g} / \mathrm{mL})$. That fact could elucidate the predominant mechanism of killing bacteria and the higher efficiency obtained using AgNPs coated Janus microbots. Thus, the effective water disinfection carried out by AgNPs coated Janus microbots may be caused by: (i) the $\mathrm{Ag}^{+}$ions efficiently released from AgNPs attached on the microbots into bacteria cytoplasma since bacteria membrane presents local lower $\mathrm{pH}^{36}$ and (ii) the enhancement of the killing contact of immobilized AgNPs with bacteria and the self-mixing provoked by self-propelled microbots. The level of $\mathrm{Ag}^{+}$allowed by the World Health Organization (WHO) in drinking water is $0.005 \mu \mathrm{g} / \mathrm{mL}$ for normal drinking water. If it is know bacteria are present in the drinking water sources, the WHO advises a $\mathrm{Ag}^{+}$level of upto $0.1 \mu \mathrm{g} / \mathrm{mL}$. 
The presented microswimmer releases $\mathrm{Ag}^{+}$concentrations, with or without bacteria, well within this range, making them an ideal choice for water remediation.

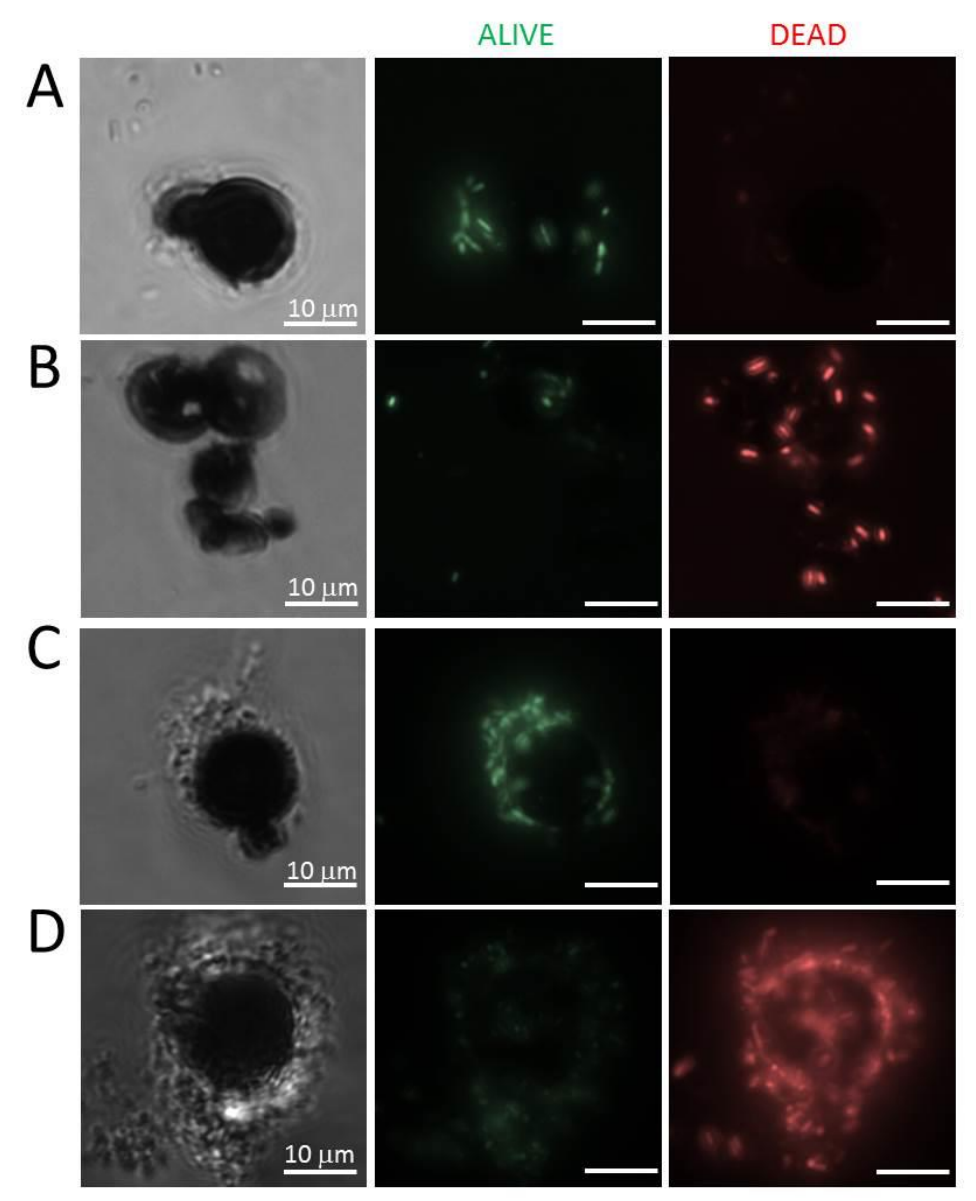

Figure 4. Captured bacteria onto AgNPs-coated Janus microbots after bactericidal assay. Images corresponding to captured bacteria in PBS on (A) Au/Fe/Mg Janus microparticles (JP) and (B) AgNPs coated Janus microbots (AgNPs-JP). Images corresponding to captured bacteria in water on (C) $\mathrm{Au} / \mathrm{Fe} / \mathrm{Mg}$ Janus microparticles (JP) and (D) AgNPs coated Janus microbots (AgNPs-JP). (Note all images have same scale bar).

As it has been previously demonstrated that bacteria display preferential adhesion to metals ${ }^{47,48}$ and the negative charge of their cell wall favors their interactions with positive charged surfaces by van der Waals and electrostatic forces. ${ }^{52,53}$ Thus, these interactions promote, in this particular case, the adhesion to the Au cap where the AgNPs are attached (Figure 4 and 5). The bacteria 
adhesion to the AgNPs modified Au surface and the low z-potential that the synthesized AgNPs (PVP-capped AgNPs, z-potential $=-10 \mathrm{mV})^{54}$ favor the mortality of bacteria and their posterior removal from the solution by the removal of the residual microbot structures after the antibacterial assay. Figure 4 confirms the adhesion of bacteria in water and PBS to the metal surfaces of the AgNPs coated Janus microbots (B and $\mathbf{D}$ ), but also on the surface of the $\mathrm{Au} / \mathrm{Fe} / \mathrm{Mg}$ Janus microparticles (A and $\mathbf{C}$ ). Bacteria attached to the non-AgNPs coated Au surface (Figure $4 \mathbf{A}$ and C) are mostly alive bacteria, as observed in the fluorescence microscope by the emission of green color. However, the bacteria attached on the AgNPs coated gold surface of the microbots are mostly dead (red color) (Figure 4 B and D).

As the microbots contain $\mathrm{Fe}$ as a sandwitched material $(\mathrm{Au} / \mathrm{Fe} / \mathrm{Mg})$ on the particle, AgNPs coated Janus microbots are capable to remove the bacteria from contaminated solutions using their magnetic properties. Figure 5A and Video S3 display a microbot externally guided using a simple permanent neodymium magnet. The microbot swims following the applied magnetic field and can also alter its direction upon changes in the magnetic field orientation similarly to other previously reported Janus micromotors. ${ }^{55,56}$ Figure 5B and Video S4 show an immobilized microbots during bactericidal assay. As it is marked by the yellow arrows, bacteria are attached on the Au surface modified with AgNPs which helps to kill bacteria and remove them from the solution. After the removal of bacteria by magnetic attraction of the microbots, they were observed by SEM (Figure 5C). SEM images confirm that the residual microbot surface is fully covered by bacteria. Attachment of bacteria to different metals has been previously reported, where guided bacteria adhesion was exploited for creating swimming biohybrids ${ }^{48}$ but here the metal cap has dual capabilities of capturing bacteria and then killing them. The magnetic properties of the cap, allow 
them to be removed from solution with the captured, dead E. coli making the microbot optimal for water purification applications.

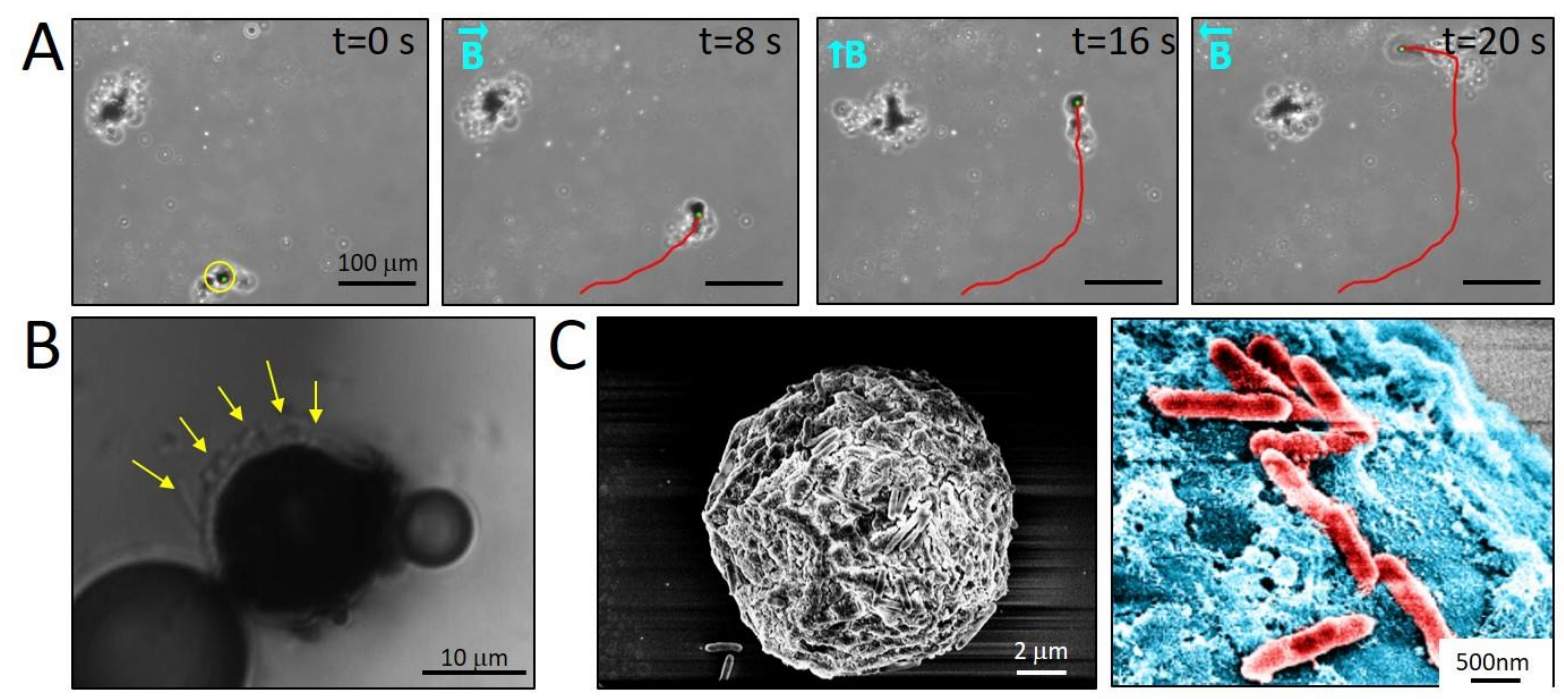

Figure 5. Magnetic and bacteria adhesion properties of AgNPs coated Janus microbots (A) Magnetic control of AgNPs coated Janus microbots using an external magnetic field. (B) Optical image of bacteria attachment on an immobilized AgNPs coated Janus microbot during bactericidal assay. (C) Left: top view of a cap from a Janus microbot after cleaning water assay and right: close view of the attached E. Coli (red) on the Au surface of Janus microbots (blue).

\section{Conclusions}

Due to the harmful disinfection by-products produced by the use of conventional disinfectant and the resistance developed by some pathogens to them, there is an urgent need to develop more effective, innovative, lower-cost, robust and safe water cleaning methods. Here, we demonstrated that Janus microbots decorated with silver nanoparticles are an efficient bactericidal tool for water disinfection. Janus microbots are self-propelled in water and contain a layer of iron which can be used to control their swimming and to remove them after their use from the clean solution using external magnets. Such controls can help to achieve targeted attack of microbots on specific sites and to avoid additional contaminats in solution. The high antibacterial efficiency of microbots can 
be explained by mainly two properties of the microbots: (i) active motion of microbots, which let the microbots to travel around and improve the chances of the contact of surface decorated AgNPs with the bacteria and also their self-propulsion can increase diffusion of released $\mathrm{Ag}^{+}$ions from the AgNPs, and (ii) the capacity of attachment of bacteria on the AgNPs coated Janus microbots after contact which provokes a major effect and speed in killing bacteria by the selective $\mathrm{Ag}^{+}$ released. We have proved the successful combination of active systems and nanomaterials to develop new micromotors for the cleaning of waterborne bacteria from contaminated water. Future work will be carried out using pathogenic bacteria in contaminated drinking water. This work opens real posibilities to develop novel micro- and nanomachines for demanded energy and environmental applications.

\section{ACKNOWLEDGMENTS}

The results leading to the publication have received financial support from the European Research Council for the European Union's Seventh Framework Programme (FP7/2007-2013)/ ERC Grant Agreement 311529 (Lab-in-a-tube and Nanorobotics biosensors), the Alexander von Humboldt

Foundation (DV) and the Grassroots Initiative funding from the Max Planck Institute for Intelligent Systems.

\section{REFERENCES}

(1) Shannon, M. A.; Bohn, P. W.; Elimelech, M.; Georgiadis, J. G.; Marinas, B. J.; Mayes, A. M. Science and technology for water purification in the coming decades. Nature 2008, 452, 301-310.

(2) Li, Q.; Mahendra, S.; Lyon, D. Y.; Brunet, L.; Liga, M. V.; Li, D.; Alvarez, P. J. J. Antimicrobial nanomaterials for water disinfection and microbial control: Potential applications and implications. Water Research 2008, 42, 4591-4602.

(3) Koivunen, J.; Heinonen-Tanski, H. Inactivation of enteric microorganisms with chemical disinfectants, UV irradiation and combined chemical/UV treatments. Water Research 2005, 39, 15191526.

(4) Wolfe, R. L. Ultraviolet disinfection of potable water. Environmental science \& technology 1990, 24, 768-773. 
(5) Anpilov, A. M.; Barkhudarov, E. M.; Christofi, N.; Kop'ev, V. A.; Kossyi, I. A.; Taktakishvili, M. I.; Zadiraka, Y. Pulsed high voltage electric discharge disinfection of microbially contaminated liquids. Lett. Appl. Microbiol. 2002, 35, 90-94.

(6) Cappelli, G.; Riccardi, M.; Perrone, S.; Bondi, M.; Ligabue, G.; Albertazzi, A. Water treatment and monitor disinfection. Hemodialysis International 2006, 10, S13-S18.

(7) Hoff, J. C.; Akin, E. W. Microbial resistance to disinfectants: mechanisms and significance. Environ. Health Perspect. 1986, 69, 7-13.

(8) Krasner, S. W. Occurrence of a new generation of disinfection byproducts. Environ. Sci. Technol. 2006, 40, 7175-7185.

(9) Li, J.; Rozen, I.; Wang, J. Rocket Science at the Nanoscale. ACS Nano 2016, 10, 5619-

5634.

(10) Orozco, J.; Jurado-Sánchez, B.; Wagner, G.; Gao, W.; Vazquez-Duhalt, R.; Sattayasamitsathit, S.; Galarnyk, M.; Cortés, A.; Saintillan, D.; Wang, J. Bubble-Propelled Micromotors for Enhanced Transport of Passive Tracers. Langmuir : the ACS journal of surfaces and colloids 2014, 30 , 5082-5087.

(11) Orozco, J.; Cheng, G.; Vilela, D.; Sattayasamitsathit, S.; Vazquez-Duhalt, R.; ValdésRamírez, G.; Pak, O. S.; Escarpa, A.; Kan, C.; Wang, J. Micromotor-Based High-Yielding Fast Oxidative Detoxification of Chemical Threats. Angewandte Chemie International Edition 2013, 52, 13276-13279.

(12) Soler, L.; Magdanz, V.; Fomin, V. M.; Sanchez, S.; Schmidt, O. G. Self-Propelled Micromotors for Cleaning Polluted Water. ACS Nano 2013, 7, 9611-9620.

(13) Guix, M.; Mayorga-Martinez, C. C.; Merkoçi, A. Nano/Micromotors in (Bio)chemical Science Applications. Chem. Rev. 2014, 114, 6285-6322.

(14) Soler, L.; Sanchez, S. Catalytic nanomotors for environmental monitoring and water remediation. Nanoscale 2014, 6, 7175-7182.

(15) Singh, V. V.; Wang, J. Nano/micromotors for security/defense applications. A review. Nanoscale 2015, 7, 19377-19389.

(16) Moo, J. G. S.; Pumera, M. Chemical Energy Powered Nano/Micro/Macromotors and the Environment. Chemistry - A European Journal 2015, 21, 58-72.

(17) Parmar, J.; Vilela, D.; Pellicer, E.; Esqué-de los Ojos, D.; Sort, J.; Sánchez, S. Reusable and Long-Lasting Active Microcleaners for Heterogeneous Water Remediation. Advanced Functional Materials 2016, 26, 4152-4161.

(18) Mushtaq, F.; Guerrero, M.; Sakar, M. S.; Hoop, M.; Lindo, A. M.; Sort, J.; Chen, X.; Nelson, B. J.; Pellicer, E.; Pane, S. Magnetically driven Bi2O3/BiOCl-based hybrid microrobots for photocatalytic water remediation. Journal of Materials Chemistry A 2015, 3, 23670-23676.

(19) Teo, W. Z.; Zboril, R.; Medrik, I.; Pumera, M. Back Cover: Fe0 Nanomotors in Ton Quantities (1020 Units) for Environmental Remediation (Chem. Eur. J. 14/2016). Chemistry - A European Journal 2016, 22, 5020-5020.

(20) Wani, O. M.; Safdar, M.; Kinnunen, N.; Jänis, J. Dual Effect of Manganese Oxide Micromotors: Catalytic Degradation and Adsorptive Bubble Separation of Organic Pollutants. Chemistry - A European Journal 2016, 22, 1244-1247.

(21) Li, J.; Singh, V. V.; Sattayasamitsathit, S.; Orozco, J.; Kaufmann, K.; Dong, R.; Gao, W.; Jurado-Sanchez, B.; Fedorak, Y.; Wang, J. Water-Driven Micromotors for Rapid Photocatalytic Degradation of Biological and Chemical Warfare Agents. ACS nano 2014, 8, 11118-11125.

(22) Guix, M.; Orozco, J.; García, M.; Gao, W.; Sattayasamitsathit, S.; Merkoçi, A.; Escarpa, A.; Wang, J. Superhydrophobic Alkanethiol-Coated Microsubmarines for Effective Removal of Oil. ACS nano 2012, 6, 4445-4451.

(23) Orozco, J.; Pan, G.; Sattayasamitsathit, S.; Galarnyk, M.; Wang, J. Micromotors to capture and destroy anthrax simulant spores. Analyst 2015, 140, 1421-1427. 
(24) Orozco, J.; Mercante, L. A.; Pol, R.; Merkoci, A. Graphene-based Janus micromotors for the dynamic removal of pollutants. Journal of Materials Chemistry A 2016, 4, 3371-3378.

(25) Seah, T. H.; Zhao, G.; Pumera, M. Surfactant Capsules Propel Interfacial Oil Droplets: An Environmental Cleanup Strategy. ChemPlusChem 2013, 78, 395-397.

(26) Gao, W.; Feng, X.; Pei, A.; Gu, Y.; Li, J.; Wang, J. Seawater-driven magnesium based Janus micromotors for environmental remediation. Nanoscale 2013, 5, 4696-4700.

(27) Singh, V. V.; Martin, A.; Kaufmann, K.; D. S. de Oliveira, S.; Wang, J. Zirconia/Graphene Oxide Hybrid Micromotors for Selective Capture of Nerve Agents. Chemistry of Materials 2015, 27, 81628169.

(28) Mou, F.; Pan, D.; Chen, C.; Gao, Y.; Xu, L.; Guan, J. Magnetically Modulated Pot-Like MnFe2O4 Micromotors: Nanoparticle Assembly Fabrication and their Capability for Direct Oil Removal. Advanced Functional Materials 2015, 25, 6173-6181.

(29) Vilela, D.; Parmar, J.; Zeng, Y.; Zhao, Y.; Sánchez, S. Graphene-Based Microbots for Toxic Heavy Metal Removal and Recovery from Water. Nano Letters 2016, 16, 2860-2866.

(30) Hoop, M.; Shen, Y.; Chen, X.-Z.; Mushtaq, F.; Iuliano, L. M.; Sakar, M. S.; Petruska, A.; Loessner, M. J.; Nelson, B. J.; Pané, S. Magnetically Driven Silver-Coated Nanocoils for Efficient Bacterial Contact Killing. Advanced Functional Materials 2016, 26, 1063-1069.

(31) Delezuk, J. A. M.; Ramirez-Herrera, D. E.; Esteban-Fernandez de Avila, B.; Wang, J. Chitosan-based water-propelled micromotors with strong antibacterial activity. Nanoscale 2017, 9 , 2195-2200.

(32) Ge, Y.; Liu, M.; Liu, L.; Sun, Y.; Zhang, H.; Dong, B. Dual-Fuel-Driven Bactericidal Micromotor. Nano-Micro Letters 2016, 8, 157-164.

(33) Castellano, J. J.; Shafii, S. M.; Ko, F.; Donate, G.; Wright, T. E.; Mannari, R. J.; Payne, W. G.; Smith, D. J.; Robson, M. C. Comparative evaluation of silver-containing antimicrobial dressings and drugs. International Wound Journal 2007, 4, 114-122.

(34) Chen, X.; Schluesener, H. J. Nanosilver: A nanoproduct in medical application. Toxicol. Lett. 2008, 176, 1-12.

(35) Quadros, M. E.; Marr, L. C. Silver Nanoparticles and Total Aerosols Emitted by Nanotechnology-Related Consumer Spray Products. Environmental science \& technology 2011, 45, 10713-10719.

(36) Xiu, Z.-m.; Zhang, Q.-b.; Puppala, H. L.; Colvin, V. L.; Alvarez, P. J. J. Negligible ParticleSpecific Antibacterial Activity of Silver Nanoparticles. Nano Letters 2012, 12, 4271-4275.

(37) Lara, H. H.; Ayala-Núñez, N. V.; Ixtepan Turrent, L. d. C.; Rodríguez Padilla, C. Bactericidal effect of silver nanoparticles against multidrug-resistant bacteria. World Journal of Microbiology and Biotechnology 2010, 26, 615-621.

(38) Morones-Ramirez, J. R.; Winkler, J. A.; Spina, C. S.; Collins, J. J. Silver Enhances Antibiotic Activity Against Gram-negative Bacteria. Science translational medicine 2013, 5, 190ra181-190ra181.

(39) Rai, M.; Yadav, A.; Gade, A. Silver nanoparticles as a new generation of antimicrobials. Biotechnology Advances 2009, 27, 76-83.

(40) Sondi, I.; Salopek-Sondi, B. Silver nanoparticles as antimicrobial agent: a case study on E. coli as a model for Gram-negative bacteria. Journal of Colloid and Interface Science 2004, 275, 177-182.

(41) Kemper, M. A.; Urrutia, M. M.; Beveridge, T. J.; Koch, A. L.; Doyle, R. J. Proton motive force may regulate cell wall-associated enzymes of Bacillus subtilis. J. Bacteriol. 1993, 175, 5690-5696.

(42) Koch, A. L. The pH in the neighborhood of membranes generating a protonmotive force. J. Theor. Biol. 1986, 120, 73-84.

(43) Xiu, Z.-M.; Ma, J.; Alvarez, P. J. J. Differential Effect of Common Ligands and Molecular Oxygen on Antimicrobial Activity of Silver Nanoparticles versus Silver lons. Environmental science \& technology 2011, 45, 9003-9008. 
(44) Mou, F.; Chen, C.; Ma, H.; Yin, Y.; Wu, Q.; Guan, J. Self-Propelled Micromotors Driven by the Magnesium-Water Reaction and Their Hemolytic Properties. Angewandte Chemie International Edition 2013, 52, 7208-7212.

(45) Mou, F.; Chen, C.; Zhong, Q.; Yin, Y.; Ma, H.; Guan, J. Autonomous Motion and Temperature-Controlled Drug Delivery of $\mathrm{Mg} / \mathrm{Pt}$-Poly( $\mathrm{N}$-isopropylacrylamide) Janus Micromotors Driven by Simulated Body Fluid and Blood Plasma. ACS Applied Materials \& Interfaces 2014, 6, 9897-9903.

(46) Agnihotri, S.; Mukherji, S.; Mukherji, S. Immobilized silver nanoparticles enhance contact killing and show highest efficacy: elucidation of the mechanism of bactericidal action of silver. Nanoscale 2013, 5, 7328-7340.

(47) Tuson, H. H.; Weibel, D. B. Bacteria-surface interactions. Soft Matter 2013, 9, 4368-

4380.

(48) Stanton, M. M.; Simmchen, J.; Ma, X.; Miguel-López, A.; Sánchez, S. Biohybrid Janus Motors Driven by Escherichia coli. Advanced Materials Interfaces 2016, 3, 1500505-n/a.

(49) Li, L.; Gui, L.; Li, W. A colorimetric silver nanoparticle-based assay for $\mathrm{Hg}(\mathrm{II})$ using lysine as a particle-linking reagent. Microchimica Acta 2015, 182, 1977-1981.

(50) Ahlberg, S.; Antonopulos, A.; Diendorf, J.; Dringen, R.; Epple, M.; Flöck, R.; Goedecke, W.; Graf, C.; Haberl, N.; Helmlinger, J.; Herzog, F.; Heuer, F.; Hirn, S.; Johannes, C.; Kittler, S.; Köller, M.; Korn, K.; Kreyling, W. G.; Krombach, F.; Lademann, J.; Loza, K.; Luther, E. M.; Malissek, M.; Meinke, M. C.; Nordmeyer, D.; Pailliart, A.; Raabe, J.; Rancan, F.; Rothen-Rutishauser, B.; Rühl, E.; Schleh, C.; Seibel, A.; Sengstock, C.; Treuel, L.; Vogt, A.; Weber, K.; Zellner, R. PVP-coated, negatively charged silver nanoparticles: A multi-center study of their physicochemical characteristics, cell culture and in vivo experiments. Beilstein Journal of Nanotechnology 2014, 5, 1944-1965.

(51) Edberg, S. C.; Rice, E. W.; Karlin, R. J.; Allen, M. J. Escherichia coli: the best biological drinking water indicator for public health protection. Journal of Applied Microbiology 2000, 88, 106S$116 \mathrm{~S}$.

(52) Jucker, B. A.; Harms, H.; Zehnder, A. J. Adhesion of the positively charged bacterium Stenotrophomonas (Xanthomonas) maltophilia 70401 to glass and Teflon. Journal of Bacteriology 1996, $178,5472-5479$.

(53) Harkes, G.; Feijen, J.; Dankert, J. Adhesion of Escherichia coli on to a series of poly(methacrylates) differing in charge and hydrophobicity. Biomaterials 1991, 12, 853-860.

(54) El Badawy, A. M.; Silva, R. G.; Morris, B.; Scheckel, K. G.; Suidan, M. T.; Tolaymat, T. M. Surface Charge-Dependent Toxicity of Silver Nanoparticles. Environmental Science \& Technology 2011, 45, 283-287.

(55) Baraban, L.; Makarov, D.; Streubel, R.; Mönch, I.; Grimm, D.; Sanchez, S.; Schmidt, O. G. Catalytic Janus Motors on Microfluidic Chip: Deterministic Motion for Targeted Cargo Delivery. ACS nano 2012, 6, 3383-3389.

(56) Liu, L.; Liu, M.; Su, Y.; Dong, Y.; Zhou, W.; Zhang, L.; Zhang, H.; Dong, B.; Chi, L. Tadpolelike artificial micromotor. Nanoscale 2015, 7, 2276-2280. 